\title{
Colossal nonlinear optical magnetoelectric effects in multiferroic $\mathrm{Bi}_{2} \mathrm{FeCrO}_{6}$
}

\author{
Sheng $\mathrm{Ju}^{1,2, \mathrm{a})}$ and Guang-Yu Guo ${ }^{1, \mathrm{~b})}$ \\ ${ }^{1}$ Department of Physics and Center of Theoretical Sciences, National Taiwan University, Taipei 106, Taiwan \\ ${ }^{2}$ Department of Physics, Suzhou University, Suzhou 215006, People's Republic of China
}

(Received 18 March 2008; accepted 21 April 2008; published online 21 May 2008)

\begin{abstract}
Within density functional theory with the generalized gradient approximation plus on-site Coulomb repulsion method, we investigate magnetic ordering dependence of linear and nonlinear optical responses in multiferroic $\mathrm{Bi}_{2} \mathrm{FeCrO}_{6}$. We find that in spite of the minor difference in the linear dielectric function between ferrimagnetic state and ferromagnetic state, the second-harmonic generation susceptibility and its related linear electro-optical coefficient dramatically change. Such colossal magnetoelectric effects come mainly from the enhanced contrast in the double-photon resonance absorption between two magnetic configurations, and will open a direction in the application of conventional multiferroics via nonlinear magnetoelectric effect. (C) 2008 American Institute of Physics. [DOI: 10.1063/1.2927474]
\end{abstract}

Materials with strongly coupled magnetic and electronic degrees of freedom provides both challenges for fundamental many-body physics and advanced functional materials. One such coupling in the itinerant limit is the interplay between ferromagnetism and charge order in the colossal magnetoresistive manganites. In the limit of localized charge, effects of strong coupling are more subtle, and chiefly manifested through a giant magnetodielectric (MD) response. Recently, multiferrroics, where both electric and magnetic orderings coexist in the same phase, have attracted great interest. ${ }^{1-8}$ Such systems are rare in nature. $\mathrm{Bi}^{3+}$ based multiferroics is one of such systems and has been extensively studied. ${ }^{6} \mathrm{Com}-$ pared with the improper ferroelectrics, ${ }^{3} \mathrm{Bi}^{3+}$ based multiferroics shows a much higher Curie temperature and stronger electric polarization. For example, $\mathrm{BiFeO}_{3}$ is ferroelectric with the Curie temperature of $1123 \mathrm{~K}$ and electric polarization of $90-100 \mu \mathrm{C} / \mathrm{cm}^{2}{ }^{7}$ However, since the magnetic and ferroelectric orderings independently origin in these materials, it is believed that the magnetoelectric (ME) coupling between two orders is very weak. On the other hand, as recently suggested by Tokura, ${ }^{5}$ these conventional polar magnets can generally show very interesting linear and nonlinear optical properties arising from the ME response in the optical frequency region, yet the coupling between electric polarization $P$ and magnetization $M$ at their electronic ground state appears to remain very small.

In this letter, we focus on nonlinear optical ME (NOME) effects of a highly distorted double-perovskite $\mathrm{Bi}_{2} \mathrm{FeCrO}_{6}$, with a predicted polarization of $80 \mu \mathrm{C} / \mathrm{cm}^{2}$ comparable with $\mathrm{BiFeO}_{3}$ and a magnetization $160 \mathrm{emu} / \mathrm{cm}^{3}$ due to its ferrimagnetic (FI) ground state. ${ }^{9}$ Although present experiments have shown a little decreased magnetization (due to the antisite disorder), ${ }^{10}$ for the coexistence of large and robust $P$ and $M, \mathrm{Bi}_{2} \mathrm{FeCrO}_{6}$ shows great advantages over other multiferroics. Previous $a b$ initio studies of electronic ground state show very weak ME coupling, ${ }^{9}$ the results of which are similar with the observation in $\mathrm{BiFeO}_{3}$ (Ref. 11) and $\mathrm{Bi}_{2} \mathrm{NiMnO}_{6} \cdot{ }^{12}$ As revealed in the following, high energy NOME coupling in $\mathrm{Bi}_{2} \mathrm{FeCrO}_{6}$, on the other hand, is colossal. With full relaxed structure, the calculation is performed

\footnotetext{
${ }^{\text {a)} E l e c t r o n i c ~ m a i l: ~ j u s h e n g @ s u d a . e d u . c n . ~}$

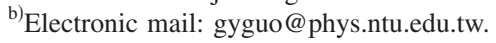

on the linear dielectric function, second-harmonic generation (SHG) susceptibilities and linear electro-optic (LEO) coefficients. FI state with antiparallel and ferromagnetic (FM) state with parallel $\mathrm{Fe}^{3+}(S=5 / 2)$ and $\mathrm{Cr}^{3+}(S=3 / 2)$ spins are compared. A sizeable difference in static SHG (170.4\%) susceptibility and LEO coefficient $(177.8 \%)$ between FI and FM sates are found. The predicted NOME effect is general in $\mathrm{Bi}^{3+}$ based multiferroics and will open a novel direction in the application of multiferroics.

Our $a b$ initio calculations are performed using the highly accurate full-potential projector augmented wave (PAW) method, ${ }^{13}$ as implemented in the Vienna $a b$ initio Simulation Package (VASP) package. ${ }^{14}$ They are based on density functional theory with the generalized gradient approximation (GGA). The on-site Coulomb interaction is included in the $\mathrm{GGA}+U$ approach $^{15}$ with $U=5 \mathrm{eV}$ and $J=0.9 \mathrm{eV}$ for both Fe and Cr $3 d$ electrons. ${ }^{9}$ A large plane-wave cutoff of $500 \mathrm{eV}$ is used throughout, and the convergence criteria for energy is $10^{-6} \mathrm{eV}$. PAW potentials are used to describe the electron-ion interaction, with 15 valence electrons for $\mathrm{Bi}$ $\left(5 d^{10} 6 s^{2} 6 p^{3}\right), 14$ for $\mathrm{Fe}\left(3 p^{6} 3 d^{6} 4 s^{2}\right), 12$ for $\mathrm{Cr}\left(3 p^{6} 3 d^{5} 4 s^{1}\right)$, and 6 for $\mathrm{O}\left(2 s^{2} p^{4}\right)$. Brillouin zone intergrations are performed with tetrahedron method in a $10 \times 10 \times 10$ Monkhorst-Pack $k$-point mesh centered at $\Gamma{ }^{16}$ The full relaxed crystal structure displayed in Table I shows a $R 3$ rhombohedral symmetry, in good agreement with recent experiments ${ }^{10}$ as well as previous calculations. ${ }^{9}$ Also, total energies indicate that FI state is the ground state, $0.0299 \mathrm{eV} /$ f.u. lower than FM state.

The optical properties are calculated based on the independent-particle approximation. ${ }^{17}$ The imaginary part of

TABLE I. Structure parameters for $R 3$ rhombohedral $\mathrm{Bi}_{2} \mathrm{FeCrO}_{6}$ with $a=0.5678 \mathrm{~nm}$ and $\alpha=59.28^{\circ}$.

\begin{tabular}{ccccc}
\hline \hline Site & Wyckoff position & $x$ & $y$ & $z$ \\
\hline $\mathrm{Bi}_{1}$ & $1 a$ & 0.0000 & 0.0000 & 0.0000 \\
$\mathrm{Bi}_{2}$ & $1 a$ & 0.5034 & 0.5034 & 0.5034 \\
$\mathrm{Fe}$ & $1 a$ & 0.7255 & 0.7255 & 0.7255 \\
$\mathrm{Cr}$ & $1 a$ & 0.2199 & 0.2199 & 0.2199 \\
$\mathrm{O}_{1}$ & $3 b$ & 0.5407 & 0.9415 & 0.3914 \\
$\mathrm{O}_{2}$ & $3 b$ & 0.0400 & 0.8970 & 0.4406 \\
\hline \hline
\end{tabular}




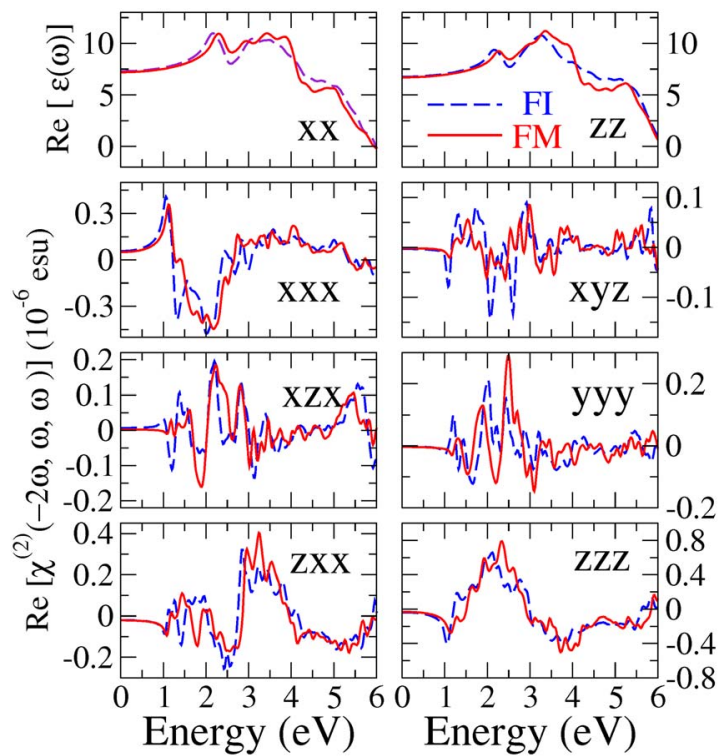

FIG. 1. (Color online) Real part of $\epsilon(\omega)$ and $\chi^{(2)}$ of FI (blue dashed line) and FM (red solid line) $\mathrm{Bi}_{2} \mathrm{FeCrO}_{6}$.

the dielectric function due to direct interband transitions is given by Fermi golden rule and the real part is obtained by a Kramer-Kronig transformation. In the mean time, the imaginary part of the second-order optical susceptibility due to direct interband transitions is given by the contribution from both the so-called virtual-electron process and the virtualhole process. The real part of the second-order optical susceptibility is then obtained by a Kramer-Kronig transformation. In the present calculations, the $\delta$ functions ${ }^{17}$ are approximated by a Gaussian function with $\Gamma=0.1 \mathrm{eV}$. The dense grid of $k$ points $16 \times 16 \times 16$ with total irreducible 690 $k$ points, 20 bands/atom, and the $50 \mathrm{eV}$ of the maximum energy in the integrals of real part of $\epsilon$ and $\chi^{(2)}$ are used to guarantee the accuracy of the present optical calculations. For the $R 3$ symmetry, among the three components of linear dielectric function $\epsilon_{x x}, \epsilon_{y y}$ and $\epsilon_{z z}, \epsilon_{x x}=\epsilon_{y y}$. While for the SHG susceptibilities, there are 13 nonvanishing components, ${ }^{18}$ with $\quad \chi_{x x x}^{(2)}=-\chi_{x y y}^{(2)}=-\chi_{y y x}^{(2)}, \quad \chi_{z y z}^{(2)}=-\chi_{y z x}^{(2)}, \quad \chi_{x z x}^{(2)}$ $=\chi_{y y z}^{(2)}, \chi_{y y y}^{(2)}=-\chi_{x x y}^{(2)}=-\chi_{y x x}^{(2)}, \chi_{z x x}^{(2)}=\chi_{z y y}^{(2)}$, and $\chi_{z z z}^{(2)^{2} z}$. As shown in Fig. 1, there is little difference in $\epsilon$ between FI and FM states. However, SHG coefficients display great contrast. In particular, the relative change in the static SHG susceptibilities and LEO coefficients can reach as high as $170.4 \%$ and $177.8 \%$, respectively (see Table II). Therefore, the LEO effect, which describes the change in the refraction under an external dc electric field, will also be magnetic field dependent in $\mathrm{Bi}_{3} \mathrm{FeCrO}_{6}$. On the other hand, the static (electronic part) $\epsilon_{x x}$ and $\epsilon_{z z}$ hardly change (less than $2 \%$ ). In fact, experiments have demonstrated that in most of $\mathrm{Bi}^{3+}$ based multiferroics $\epsilon(0)$ (ionic+electronic) is almost unsusceptible to magnetic ordering and MD effect is extremely small, e.g., in $\mathrm{BiMnO}_{3}$ less than $0.6 \%$ change in $\epsilon(0)$ at $90 \mathrm{kOe}^{8}$

To microscopically understand the above colossal NOME, we plot the absolute value of imaginary part of $\chi^{(2)}$ in contrast with $\epsilon^{\prime \prime}(\omega)$ and $\epsilon^{\prime \prime}(\omega / 2)$ (see Fig. 2). Firstly, we can find that SHG spectrum can be divided into three regions: the regime with $\hbar \omega \leqslant 2 \mathrm{eV}$, corresponding doublephoton resonance; the regime with $2 \mathrm{eV} \leqslant \hbar \omega \leqslant 3 \mathrm{eV}$, corresponding joint double-photon and single-photon resonances; and the regime beyond, related with single-photon resoDownloaded 02 Jul 2009 to 140.112.113.225. Redistribution subject
TABLE II. Calculated static dielectric constant $(\epsilon)$, second-order susceptibility $\left[\chi^{(2)}\right]\left(10^{-8} \mathrm{esu}\right)$, LEO coefficient $\left(r^{e}\right)(\mathrm{pm} / \mathrm{V})$, and corresponding optical $\mathrm{ME}$ effect in $\mathrm{Bi}_{2} \mathrm{FeCrO}_{6}$.

\begin{tabular}{cccr}
\hline \hline & $\mathrm{FI}$ & $\mathrm{FM}$ & $\mathrm{OME}$ \\
\hline$\epsilon_{x x}$ & 7.29 & 7.20 & $1.3 \%$ \\
$\epsilon_{z z}$ & 6.76 & 6.70 & $0.8 \%$ \\
$\chi_{x x x}^{(2)}$ & 5.72 & 5.04 & $13.5 \%$ \\
$\chi_{x y z}^{(2)}$ & -0.31 & -0.17 & $82.4 \%$ \\
$\chi_{x z x}^{(2)}$ & 0.73 & 0.27 & $170.4 \%$ \\
$\chi_{y y y}^{(2)}$ & -0.23 & -0.35 & $52.2 \%$ \\
$\chi_{z x x}^{(2)}$ & -1.99 & -2.10 & $5.5 \%$ \\
$\chi_{z z z}^{(2)}$ & -3.71 & -3.11 & $19.3 \%$ \\
$r_{x x x}^{e}$ & -0.90 & -0.815 & $10.4 \%$ \\
$r_{x y z}^{e}$ & 0.05 & 0.025 & $100.0 \%$ \\
$r_{x z x}^{e}$ & -0.125 & -0.045 & $177.8 \%$ \\
$r_{y y y}^{e}$ & 0.035 & 0.055 & $57.1 \%$ \\
$r_{z x x}^{e}$ & 0.34 & 0.365 & $7.4 \%$ \\
$r_{z z z}^{e}$ & 0.67 & 0.58 & $15.5 \%$ \\
\hline \hline
\end{tabular}

nance. Secondly, between FI and FM states, little difference, except the peak around $2 \mathrm{eV}$ in $\epsilon^{\prime \prime}(\omega / 2)$ is observed. However, $\chi^{\prime \prime(2)}$ significantly differs in peaks' height. In particular, for the components of $x z x, x y z$, and $y y y$, great contrast in the magnitude of first several peaks $(\hbar \omega \leqslant 2 \mathrm{eV}$, i.e., linear absorption edge) is observed. This also agrees with the variation of static $\chi^{(2)}$ and $r^{e}$, as listed in Table II, and reflects the enhanced contrast in double-photon resonance between FM and FI configurations.

The detailed band structure (see Fig. 3) can be summarized as follows. For both FI and FM states, the lowest bands at $-10 \mathrm{eV}$ are mainly from $\mathrm{Bi} 6 s$ states (not shown). Above these bands are the bands of the hybridized $\mathrm{O} 2 p$ and $\mathrm{Bi} 6 p$ as well as $\mathrm{Fe}$ and $\mathrm{Cr} 3 d$ states localized near Fermi level. The conduction band is of $\mathrm{Fe}$ and $\mathrm{Cr} 3 d$ and $\mathrm{O} 2 p$ states and is followed by the band of the $\mathrm{Bi} 6 p$ states at higher energy. The hybridization between $\mathrm{Bi}$ and $\mathrm{O}$ is obvious, which is the

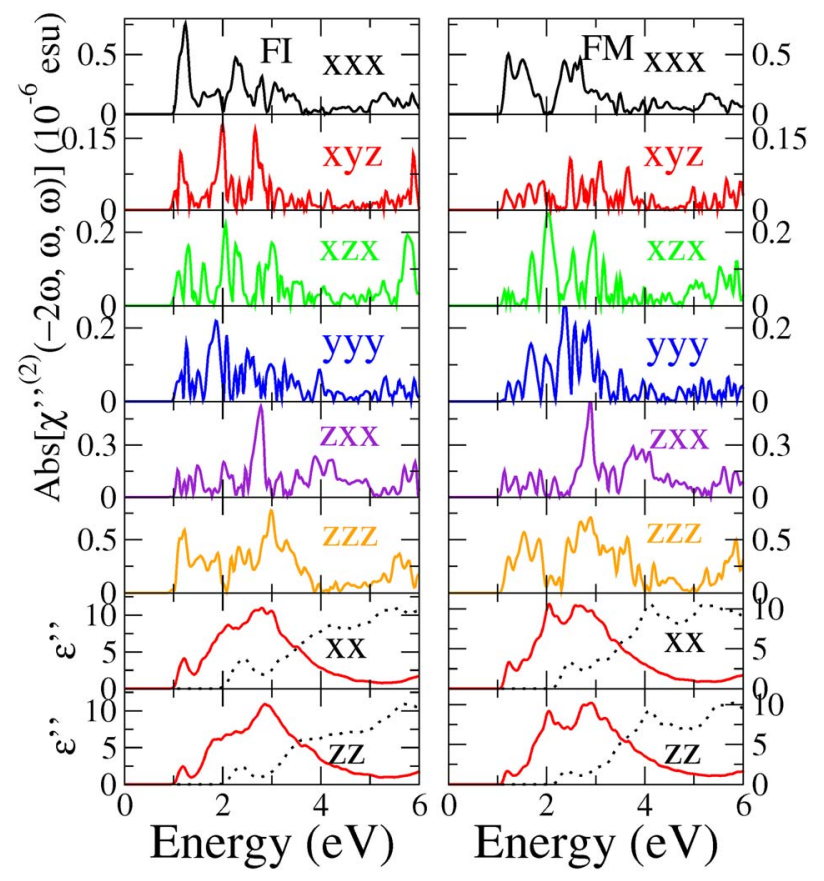

FIG. 2. (Color online) Absolute value of the imaginary part of $\chi^{(2)}$ as well as $\epsilon^{\prime \prime}(\omega)$ (black dotted line) and $\epsilon^{\prime \prime}(\omega / 2)$ (red solid line) of $\mathrm{Bi}_{2} \mathrm{FeCrO}_{6}$.

to AIP license or copyright; see http://apl.aip.org/apl/copyright.jsp 


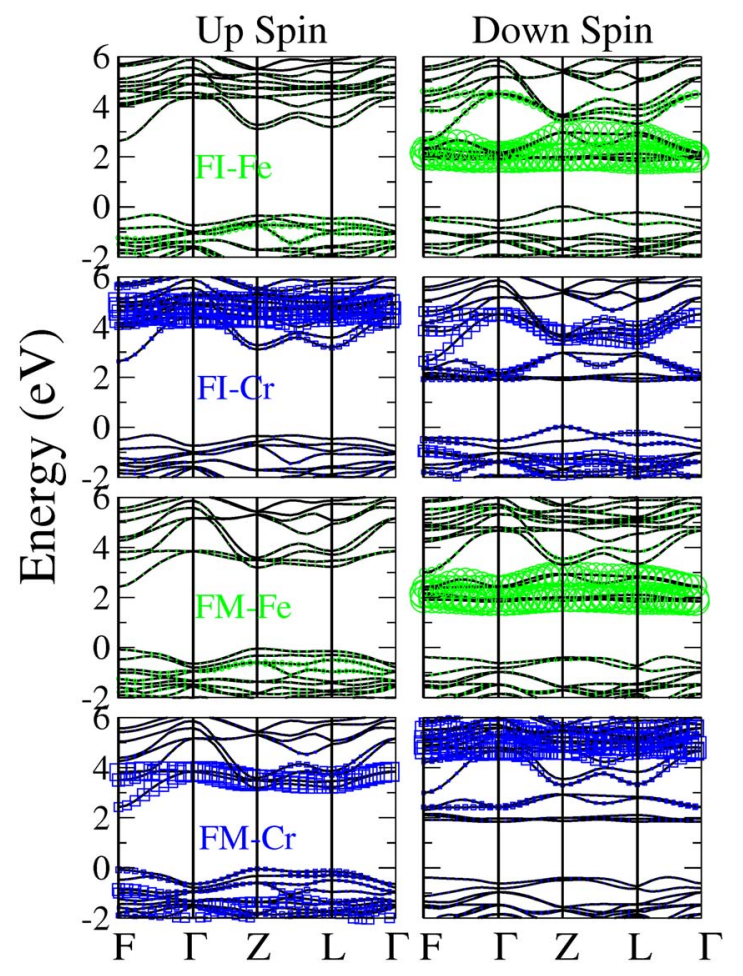

FIG. 3. (Color online) Band structure of FI (the upper two panels) and FM (the bottom two panels) $\mathrm{Bi}_{2} \mathrm{FeCrO}_{6}$. The fatness associated with the bands is proportional to $\mathrm{Fe}$ (green) or $\mathrm{Cr}$ (blue) ions.

driving force of ferroelectricity in such $A$-site driven ferroelectrics. ${ }^{6}$ When the system transits from FI to FM, the upspin channel and downspin channel of $\mathrm{Cr}$ site interchange.

It is generally believed that since linear dielectric constant of insulators is dominated by a band gap of scale several $\mathrm{eV}$, the magnitude of which is much larger than magnetic interaction. Like most of magnetic insulators, the coupling between magnetic ordering and linear dielectric constant in $\mathrm{Bi}_{3} \mathrm{FeCrO}_{6}$ is extremely weak. Even though a soft phonon mode of energy around $10 \mathrm{meV}$ is allowed in ferroelectrics, the change in static dielectric constant is still small. While the contrast of SHG susceptibilities between different magnetic orders is enhanced via double-photon resonance absorption.

To summarize, by studying the magnetic ordering dependence of linear and nonlinear optical properties in multifer- roic $\mathrm{Bi}_{3} \mathrm{FeCrO}_{6}$, we have revealed two novel nonlinear optical phenomena, i.e., magneto-SHG and magneto-LEO effects. Such NOME effects are much larger than linear MD effect and can be induced by either the change in the magnetic ordering with external magnetic field or the presence of spin fluctuations near magnetic phase transition temperature. We hope our $a b$ initio calculation will stimulate further experiments on NOME effects in various multiferroic systems.

The authors gratefully acknowledge supports from the National Natural Science Foundation of China under Grant No. 10504023, National Science Council of Taiwan, and NCTS of Taiwan.

${ }^{1}$ M. Fiebig, J. Phys. D 38, R123 (2005).

${ }^{2}$ W. Eerenstein, N. D. Mathur, and J. F. Scott, Nature (London) 442, 759 (2006).

${ }^{3}$ S.-W. Cheong and M. Mostovoy, Nat. Mater. 6, 13 (2007).

${ }^{4}$ R. Ramesh and N. A. Spaldin, Nat. Mater. 6, 21 (2007).

${ }^{5}$ Y. Tokura, J. Magn. Magn. Mater. 310, 1145 (2007).

${ }^{6}$ C. Ederer and N. A. Spaldin, Curr. Opin. Solid State Mater. Sci. 9, 128 (2006).

${ }^{7}$ J. Wang, J. B. Neaton, H. Zheng, V. Nagarajan, S. B. Ogale, B. Liu, D. Viehland, V. Vaithyanathan, D. G. Schlom, U. V. Waghmare, N. A. Spaldin, K. M. Rabe, M. Wuttig, and R. Ramesh, Science 299, 1719 (2003).

${ }^{8}$ T. Kimura, S. Kawamoto, I. Yamada, M. Azuma, M. Takano, and Y. Tokura, Phys. Rev. B 67, 180401 (2003).

${ }^{9}$ P. Baettig and N. A. Spaldin, Appl. Phys. Lett. 86, 012505 (2005); P. Baettig, C. Ederer, and N. A. Spaldin, Phys. Rev. B 72, 214105 (2005).

${ }^{10}$ R. Nechache, C. Harnagea, A. Pignolet, F. Normandin, T. Veres, L. P. Carignan, and D. Menard, Appl. Phys. Lett. 89, 102902 (2006); R. Nechache, C. Harnagea, L. P. Carignan, D. Menard, and A. Pignolet, Philos. Mag. Lett. 87, 231 (2007); M. R. Suchomel, C. I. Thomas, M. Allix, M. J. Rosseinsky, and A. M. Fogg, Appl. Phys. Lett. 90, 112909 (2007); D. H. Kim, H. N. Lee, M. D. Biegalski, and H. M. Christen, ibid. 91, 042906 (2007).

${ }^{11}$ C. Ederer and N. A. Spaldin, Phys. Rev. B 71, 060401 (2005).

${ }^{12}$ A. Ciucivara, B. Sahu, and L. Kleinman, Phys. Rev. B 76, 064412 (2007).

${ }^{13}$ P. E. Blochl, Phys. Rev. B 50, 17953 (1994).

${ }^{14}$ G. Kresse and J. Hafner, Phys. Rev. B 47, 558 (1993); 49, 14 (1994); G. Kresse and J. Furthmuller, Comput. Mater. Sci. 6, 15 (1996); G. Kresse and D. Joubert, Phys. Rev. B 59, 1758 (1999).

${ }^{15}$ A. I. Liechtenstein, V. I. Anisimov, and J. Zaane, Phys. Rev. B 52, R5467 (1995).

${ }^{16}$ P. E. Blochl, O. Jepsen, and O. K. Andersen, Phys. Rev. B 49, 16223 (1994).

${ }^{17}$ G. Y. Guo, K. C. Chu, D. S. Wang, and C. G. Duan, Phys. Rev. B 69, 205416 (2004); G. Y. Guo and J. C. Lin, Phys. Rev. B 72, 075416 (2005); ibid. 77, 049901(E) (2008).

${ }^{18}$ R. W. Boyd, Nonlinear Optics (Elsevier, Amsterdam, 2003). 